\title{
Intermittent Mixed Venous Oxygen Saturation in Pediatric Septic Shock
}

\author{
Ami P Shah, ${ }^{1 *}$ Prerna Batra ${ }^{2}$ \\ ${ }^{1}$ Department of Emergency Medicine, University of Nevada, Las Vegas School of Medicine, Las Vegas, NV,USA; \\ ${ }^{2}$ Department of Pediatrics, University College of Medical Sciences and Guru Teg Bahadur Hospital, Delhi. \\ *ami.shah@unlv.edu
}

$\mathrm{A}$ cross the globe, sepsis is the leading cause of morbidity and mortality in the pediatric population. An estimated 1.2 million cases of childhood sepsis are reported worldwide [1]. Given the global burden of disease in 2001, Surviving Sepsis Campaign (SSC) was initiated to collaborate and improve research and education for sepsis survival [2,3]. Shortly after the SSC campaign was initiated, the American College of Critical Care Medicine (ACCM) and Pediatric Advanced Life Support (PALS) guidelines recommended the use of targeted mixed venous oxygen saturation (ScvO2) therapy for Early Goal Directed Therapy (EGDT) in pediatric septic shock. Targeted ScvO2 monitoring is a critical tool for guiding therapy in children with septic shock both in the developed and developing countries [4-6]. Despite extensive use of $\mathrm{ScvO} 2$ monitoring in clinical practice, there are not many studies supporting its use. Clinical practice guidelines for management of pediatric septic shock by ACCM (2014) strongly recommended the use of targeted ScvO2 levels, a surrogate for cardiac index, as a marker of therapeutic end point for resuscitation, based on case control and cohort studies [7]. However, these recommendations are based mostly on the adult studies from developed world. Feasibility of continuous ScvO2 monitoring is question-able in resource-constrained settings of low- and middle-income countries (LMICs).

De Oliveira, et al. [8] with their randomized controlled design provided a head on comparison of continuous targeted $\mathrm{ScvO} 2>70 \%$ in children with fluid refractory shock guided monitoring ( $n=51$ in each group). The authors reported a significant reduction in mortality in children with targeted $\mathrm{ScvO} 2$ therapy with numbers needed to treat (NNT) of 3.6. The study provided a premise for use of ScvO2 monitoring in clinical practice in advanced pediatric intensive care units (PICU) in the developing world. Subsequently, Sankar, et al. [9] published a prospective cohort study of children with fluid refractory shock admitted to a PICU in India. They used intermittent ScvO2 values obtained at 1, 3 and 6 hours after initiation of therapy to guide treatment against clinical variables and lactate among controls. The catheter was placed in subclavian/internal jugular vein, and the patients in whom catheter could not be placed served as controls. The authors observed a lower mortality in $\mathrm{ScvO} 2$ group as against controls ( $33 \%$ vs 54\%; NNT $=5)$. The limitation of the study was its design with a possible selection bias but it provided a balanced approach in resource-limited set ups, where continuous monitoring was not feasible.

The 2020 SSC International guidelines support the use of advanced hemodynamic monitoring such as ScvO2, but categorize it under weak low quality evidence $[2,10]$. Jain, et al. [11] report on a randomized controlled trial targeting intermittent superior vena caval saturation (ScvO2) above $70 \%$ for EGDT in patients with pediatric septic shock, in the current issue of Indian Pediatrics. The study population included children from 1 month to 12 years of age with fluid refractory shock admitted to the PICU. The authors found a significantly lower 28-day mortality and lower new organ dysfunction in the group with $\mathrm{ScvO} 2$ targeted therapy group. There was no difference noted in the time to achieve therapeutic end points, need for organ support and length of PICU or hospital stay. The study protocol is similar to the previous study from India except that fluid refractory shock was defined as shock not responding to $40 \mathrm{~mL} / \mathrm{kg}$ of fluids as compared to $60 \mathrm{~mL} / \mathrm{kg}$ used by Sankar, et al. [9]. All these studies support the utility of continuous and intermittent ScvO2 monitoring as a cost-effective tool for improving the survival of children with sepsis.

Dr. Joseph Carcillo, whose work has been instrumental in the field of pediatric septic shock, refers $\mathrm{ScvO} 2$ as a poor man's mixed venous oxygen saturation [12]. The author warns us of the limitations of the use of ScvO2 measurements. True ScvO2 requires oxygen saturation measurement of the pulmonary artery and this value can be $2 \%$ to $8 \%$ lower than catheters placed at SVC-RA and IVC$\mathrm{RA}$ junction. High $\mathrm{ScvO} 2$ can indicate improved oxygen delivery but may also be reflective of poor oxygen consumption due to sepsis induced dysfunctional tissue perfusion and mitochondrial dysfunction $[12,13]$. In 2018, 
Goonasekera, et al. [14] studied global oxygen extraction ratio (gO2ER) as a marker of oxygen consumption in fluid refractory pediatric septic shock on a cohort of 62 children admitted to the PICU with fluid refractory shock. They concluded that $\mathrm{gO} 2 \mathrm{ER}$ of $>0.48$ with a blood lactate $>4.0$ $\mathrm{mmol} / \mathrm{L}$ and metabolic acidosis are better predictors of death as compared to $\mathrm{ScvO} 2$.

Children are not young adults. With the limited evidence available in pediatric population, the study by Jain, et al. [11] continues to support the use of intermittent targeted $\mathrm{ScvO} 2$ monitoring for improving survival in pediatric septic shock refractory to $40 \mathrm{~mL} / \mathrm{kg}$ of fluid resuscitation. However, the results mostly apply to sick children with septic shock having low $\mathrm{SvcO} 2$. With the clinical world constantly searching for more non-invasive methods, implementing the principles of EGDT using echocardiography and point of care ultrasound (POCUS) in children with septic shock may give newer insights in future $[6,14]$.

Funding: None. Competing interests: None.

\section{REFERENCES}

1. Fleischmann-Struzek C, Goldfarb DM, Schlattmann P, Schlapbach LJ, Reinhart K, Kissoon N. The global burden of paediatric and neonatal sepsis: A systematic review. Lancet Respir Med. 2018;6:223-30.

2. Weiss SL, Peters MJ, Alhazzani W, et al. Surviving sepsis campaign international guidelines for the management of septic shock and sepsis-associated organ dysfunction in children. Pediatr Crit Care Med. 2020;21:e52-e106.

3. Carcillo JA, Fields AI, American College of Critical Care Medicine Task Force Committee Members. Clinical Practice Parameters for Hemodynamic Support of Pediatric and Neonatal Patients in Septic Shock. Crit Care Med. 2002;30:1365-78.

4. Deep A, Goonasekera CD, Wang Y, Brierley J. Evolution of haemodynamics and outcome of fluid-refractory septic shock in children. Intensive Care Med. 2013;39:1602-09.

5. Brierley J, Peters MJ. Distinct hemodynamic patterns of septic shock at presentation to pediatric intensive care. Pediatrics. 2008;122:752-9.

6. Brierley J, Carcillo JA, Choong K, et al. Clinical practice parameters for hemodynamic support of pediatric and neonatal septic shock: 2007 update from the American College of Critical Care Medicine. Crit Care Med. 2009;37:666-88.

7. Davis AL, Carcillo JA, Aneja RK, et al. The American College of Critical Care Medicine clinical practice parameters for hemodynamic support of pediatric and neonatal septic shock: Executive summary. Pediatr Crit Care Med. 2017;18:884-90.

8. de Oliveira CF, de Oliveira DS, Gottschald AF, et al. ACCM/ PALS haemodynamic support guidelines for paediatric septic shock: An outcomes comparison with and without monitoring central venous oxygen saturation. Intensive Care Med. 2008;34:1065-75.

9. Sankar J, Sankar MJ, Suresh CP, Dubey NK, Singh A. Early goal-directed therapy in pediatric septic shock: Comparison of outcomes "with" and "without" intermittent superior venacaval oxygen saturation monitoring: A prospective cohort study. Pediatr Crit Care Med. 2014;15:157.

10. Weiss SL, Peters MJ,Alhazzani W, et al. Executive Summary: Surviving Sepsis Campaign International Guide-lines for the Management of Septic Shock and Sepsis-Associated Organ Dysfunction in Children. Pediatr Crit Care Med. 2020;21: 186-95.

11. Jain P, Rameshkumar R, Satheesh P, Mahadevan S. Early goaldirected therapy with and without intermittent superior vena cava oxygen saturation monitoring in pediatric septic shock: A randomized controlled trial. Indian Pediatr. 2021. May;58: 1124-30.

12. de Oliveira CF, Troster EJ, Carcillo JA. A beneficial role of central venous oxygen saturation-targeted septic shock management in children: Follow the pediatric story, not only the adult story. Pediatr Crit Care Med. 2014;15:380-2.

13. Goonasekera CDA, Carcillo JA, Deep A. Oxygen delivery and oxygen consumption in pediatric fluid refractory septic shock during the first $42 \mathrm{~h}$ of therapy and their relationship to 28-day outcome. Front Pediatr. 2018;6:314.

14. Lanspa MJ, Burk RE, Wilson EL, Hirshberg EL, Grissom CK, Brown SM. Echocardiogram-guided resuscitation versus early goal-directed therapy in the treatment of septic shock: A randomized, controlled, feasibility trial. J Intensive Care. 2018;6:50-3. 